\title{
HIGH RESOLUTION 36 GHz IMAGING OF THE SUPERNOVA REMNANT OF SN 1987A
}

\author{
T. M. Potter ${ }^{1}$, L. Staveley-Smith ${ }^{1,4}$, C.-Y. NG ${ }^{2}$, Lewis Ball ${ }^{3}$, B. M. Gaensler ${ }^{2,5}$, M. J. Kesteven ${ }^{3}$, R. N. Manchester ${ }^{3}$, \\ A. K. TZIOUMIS ${ }^{3}$, AND G. ZANARDO ${ }^{1}$ \\ ${ }^{1}$ International Centre for Radio Astronomy Research (ICRAR), School of Physics M468, The University of Western Australia, Crawley WA 6009, Australia; \\ T.Potter@aip.org.au \\ ${ }^{2}$ Sydney Institute for Astronomy (SIfA), School of Physics, The University of Sydney, Sydney NSW 2006, Australia \\ ${ }^{3}$ Australia Telescope National Facility (ATNF), CSIRO, P.O. Box 76, Epping NSW 1710, Australia \\ Received 2009 June 24; accepted 2009 September 10; published 2009 October 9
}

\begin{abstract}
The aftermath of supernova (SN) 1987A continues to provide spectacular insights into the interaction between an SN blastwave and its circumstellar environment. We here present $36 \mathrm{GHz}$ observations from the Australia Telescope Compact Array of the radio remnant of SN 1987A. These new images, taken in 2008 April and 2008 October, substantially extend the frequency range of an ongoing monitoring and imaging program conducted between 1.4 and $20 \mathrm{GHz}$. Our $36.2 \mathrm{GHz}$ images have a diffraction-limited angular resolution of $0{ }^{\prime}$. $3-0.4$, which covers the gap between high resolution, low dynamic range VLBI images of the remnant and low resolution, high dynamic range images at frequencies between 1 and $20 \mathrm{GHz}$. The radio morphology of the remnant at $36 \mathrm{GHz}$ is an elliptical ring with enhanced emission on the eastern and western sides, similar to that seen previously at lower frequencies. Model fits to the data in the Fourier domain show that the emitting region is consistent with a thick inclined torus of mean radius $00^{\prime} 85$, and a 2008 October flux density of $27 \pm 6 \mathrm{mJy}$ at $36.2 \mathrm{GHz}$. The spectral index for the remnant at this epoch, determined between $1.4 \mathrm{GHz}$ and $36.2 \mathrm{GHz}$, is $\alpha=-0.83$. There is tentative evidence for an unresolved central source with flatter spectral index.
\end{abstract}

Key words: radio continuum: general - supernovae: general - supernovae: individual (SN 1987A) - supernova remnants

\section{INTRODUCTION}

The type IIP supernova (SN) 1987A is the most well-observed and published $\mathrm{SN}$ in existence. As the brightest $\mathrm{SN}$ seen since the invention of the telescope, it has been observed right across the electromagnetic spectrum from radio through to gamma rays. It was also the first $\mathrm{SN}$ for which we have observations of the progenitor, as well as the first and only SN thus far to have been associated with a neutrino burst event (Hirata et al. 1987; Bionta et al. 1987). Neutrino observations from SN 1987A have been used to put a firm foundation on theories of core collapse and neutron star formation (Janka 1997) as well as giving a time of core collapse at 1987 February 23, 07:35 UT. Shortly after core collapse an expanding shockwave tore through the progenitor's stellar envelope. The resulting shock breakout resulted in a UV flash that ionized material in the circumstellar environment (Luo \& McCray 1991), illuminating a central equatorial ring and two outer rings that we now know are part of a much larger hourglass structure (Sugerman et al. 2005). As yet, the mechanism for ring formation is not properly understood; however, two competing theories, the interacting winds model (Blondin \& Lundqvist 1993; Martin \& Arnett 1995; Tanaka \& Washimi 2002) and the binary merger model (Podsiadlowski et al. 2007), offer plausible mechanisms for shaping the rings. At present the shock is transiting the equatorial ring and continues to provide insights into the hydrodynamics of a unique young SN remnant as followed by X-ray, optical, and radio telescopes.

In the radio SN 1987A was imaged as soon as a few days after detection. On 1987 February 25, Turtle et al. (1987) detected emission at $843 \mathrm{MHz}$ with the Molonglo Observatory

\footnotetext{
4 Premier's Fellow in Radio Astronomy.

5 Federation Fellow, Australian Research Council.

6 CSIRO Fellow.
}

Synthesis Telescope (MOST) along with detections at 1.4, 2.3, and $8.4 \mathrm{GHz}$ by an interferometer formed between the Parkes $64 \mathrm{~m}$ and Tidbinbilla $34 \mathrm{~m}$ antennas. Emission at $843 \mathrm{MHz}$ peaked around $130 \mathrm{mJy}$ on 1987 February 26-27. Just over five days after the neutrino burst (1987 February 28), Jauncey et al. (1988) used VLBI techniques to resolve the expanding radiosphere. Continued observations saw the $\mathrm{SN}$ fade from its initial radio burst to almost undetectable levels within a few months (Turtle et al. 1987). From this point on, radio emission from SN 1987A deviated radically from the trend of typical type IIP SN light curves. Radio emission from the remnant returned in 1990 with a re-detection at $843 \mathrm{MHz}$ by MOST on July 5 (Ball et al. 2001) and the six $22 \mathrm{~m}$ antennas of the Australia Telescope Compact Array (ATCA) a month later (Staveley-Smith et al. 1992). This has been interpreted as a collision between the expanding shock front and an ionized hydrogen region about half the distance to the optical ring (Chevalier \& Dwarkadas 1995). Since then, an ongoing observing campaign (Gaensler et al. 1997; Manchester et al. 2002, 2005; Staveley-Smith et al. 2007; Gaensler et al. 2007) has followed radio emission from the developing remnant at frequencies ranging from 1.4 to $20 \mathrm{GHz}$. This saw the radio emission rise at monotonically increasing rate as the shockwave began its crossing of the ring (G. Zanardo et al. 2009 , in preparation). Such behavior puts the remnant of SN 1987A in the somewhat rare category of young and brightening SN remnants, along with the Galactic SN remnant G1.9+0.3 (Reynolds et al. 2008; Murphy et al. 2008).

High precision images of the remnant at high resolution are important for studying the morphology and evolution of the blastwave interaction. Previous estimates of the optical ring geometry (Plait et al. 1995) have shown that it is an ellipse with semimajor axis $00^{\prime} 858 \pm 0.011$ and semiminor axis $00^{\prime} 621 \pm 0.011$, thus requiring very high resolution imaging. Cameras aboard the Hubble Space Telescope (HST) are capable 
Table 1

Observing Conditions for 36.2 GHz Observations of SN 1987A

\begin{tabular}{lcc}
\hline \hline \multicolumn{1}{c}{ Date } & $\begin{array}{c}\text { Path Noise } \\
(\mu \mathrm{m})\end{array}$ & $\begin{array}{c}\text { Integration Time } \\
(\mathrm{hr})\end{array}$ \\
\hline 2008 Apr 25 & $334 \pm 133$ & 11.3 \\
2008 Oct 7 & $131 \pm 22$ & 7.4 \\
2008 Oct 8 & $88 \pm 10$ & 3.9 \\
2008 Oct 12 & $184 \pm 60$ & 9.8 \\
\hline
\end{tabular}

of imaging at $0{ }^{\prime} .03-00^{\prime \prime} 2$ pixel $^{-1}$ (McMaster \& Biretta 2008) which has enabled detailed imaging of the structure of the equatorial ring as well as the development of the reverse shock (Michael et al. 2003). Pixels on the ACIS detector aboard the Chandra X-ray Observatory register half the total encircled energy of incident photons within a circle of diameter 0'.8 (half power beam diameter (HPD)), although this can be improved to around 0'.6 HPD with subsampling techniques (Tsunemi et al. 2001). In 2007 October, Tingay et al. (2009) used an e-VLBI array formed between the ATCA, Parkes, and Mopra (22 m) antennas to image the remnant of SN 1987A at $1.4 \mathrm{GHz}$ with a restoring beam of $85 \times 168$ mas. However, the limited range of baselines made such an array insensitive to details larger than 0.4 . In the radio the ATCA is capable of resolving details as small as 0.2 at around $40 \mathrm{GHz}$, which nicely fills in the gap left by the VLBI observation and images at the lower frequencies. Imaging at higher frequencies around $100 \mathrm{GHz}$ is also possible, although this capability is enable to for only five of the six antennas, cutting the number of baselines by a third as well as reducing the maximum baseline length to $3 \mathrm{~km}$. This translates to roughly the same resolution (approximately 0.2 ) as the $40 \mathrm{GHz}$ band. Unfortunately at higher frequencies atmospheric opacity degrades the image with approximately an order of magnitude higher effective system temperature (Brooks 2007). With an aim to obtain the highest resolution images currently possible with the ATCA, the ongoing imaging observations of $\mathrm{Ng}$ et al. (2008) were extended to include frequencies around $36 \mathrm{GHz}$. In this paper we present the latest $36 \mathrm{GHz}$ data from the recent extension to these ongoing observations.

\section{OBSERVATIONS AND REDUCTION}

Our observations of the SN remnant were made with the $\mathrm{ATCA}^{7}$ at center frequencies of 34.88 and $37.44 \mathrm{GHz}$ on 2008 April 25 and 2008 October 7, 8, 12 using antenna configuration $6 \mathrm{~A}$ with multifrequency imaging over $2 \times 128 \mathrm{MHz}$ bandwidth in all Stokes parameters. Atmospheric phase stability at the ATCA was monitored with two $1.8 \mathrm{~m}$ antennas spaced $230 \mathrm{~m}$ apart designed to measure the path noise of a $30 \mathrm{GHz}$ signal from the geostationary Optus-B3 communications satellite. A path noise of below $300 \mu \mathrm{m}$ is considered good for millimeter-wave astronomy at the ATCA. Table 1 shows observing conditions and un-flagged uv coverage for each observation.

The 2008 April 25 observation was hampered by poor weather, consequently around $3 \mathrm{hr}$ of uv-data was discarded. The secondary flux calibrator PKS 0637-752 was observed for 2 minutes in hourly intervals and the phase calibrator PKS 0530-727 was observed for 1.5 minutes per 6 minutes integration time on source. A flux density of $3.93 \pm 0.06$ and $3.83 \pm 0.06 \mathrm{Jy}$ at 34.88 and $37.44 \mathrm{GHz}$ was obtained for the

\footnotetext{
7 The Australia Telescope Compact Array is part of the Australia Telescope which is funded by the Commonwealth of Australia for operation as a National Facility managed by CSIRO.
}

Table 2

Image Parameters for the 2008 Observations at $36.2 \mathrm{GHz}$

\begin{tabular}{lcccc}
\hline \hline Date & Robust & $\begin{array}{c}\text { Restoring Beam } \\
\left({ }^{\prime \prime}\right)\end{array}$ & $\begin{array}{c}\text { Position Angle } \\
\left({ }^{\circ}\right)\end{array}$ & $\begin{array}{c}\text { Rms Noise } \\
\left(\text { mJy beam }^{-1}\right)\end{array}$ \\
\hline 2008 Apr 25 & 0.5 & $0.34 \times 0.24$ & 45.0 & 0.10 \\
& 2.0 & $0.38 \times 0.28$ & 44.6 & 0.10 \\
2008 Oct 7-12 & 0.5 & $0.37 \times 0.22$ & -7.7 & 0.06 \\
& 2.0 & $0.43 \times 0.25$ & -8.3 & 0.06 \\
2008 Apr-Oct & 0.5 & $0.33 \times 0.24$ & -1.3 & 0.06 \\
& 2.0 & $0.38 \times 0.27$ & -1.0 & 0.06 \\
\hline
\end{tabular}

2008 October observations of PKS 0637-752 by bootstrapping an observation of the primary flux calibrator Uranus at similar elevation $\left(\approx 35^{\circ}\right)$ from the night of 2008 October 8 . The flux density of PKS 0637-752 for the 2008 April 25 observation was similarly determined to be $3.71 \pm 0.07$ and $3.61 \pm 0.08 \mathrm{Jy}$ using a later 2008 May 25 observation of both the calibrator and Uranus (at the shortest baseline). The phase center was located about $5^{\prime \prime} .5$ south of the remnant to avoid defects (Taylor et al. 1999). We used the software package Miriad (Sault et al. 1995) to perform image reduction and analysis. Continuum images were made with robust $=0.5$ weighting (Briggs 1995) as this represented the best compromise between resolution and signalto-noise ratio. Natural weighting (robust $=2.0$ ) was used in both the flux and polarization measurements in order to preserve as much signal as possible. The CLEAN algorithm (Högbom 1974; Clark 1980) was employed to deconvolve the resulting continuum images. Table 2 gives the size and position angle of the restoring beam, as well as the rms noise of the resulting image in each case.

Shown in Figure 1 are the deconvolved Stokes-I continuum images of the $36.2 \mathrm{GHz}$ observations. Figure 1(a) is the image from the 2008 April 25 observation, Figure 1(b) is the combined 2008 October image, and Figure 1(c) is the combined 2008 April and 2008 October images. Note that the approximately northsouth extensions prominent in the images are probably artifacts caused by insufficient uv coverage and increased phase errors when observing at low elevation.

For purposes of comparison, we also obtained a contemporaneous observation at $8.6 \mathrm{GHz}$ on 2008 October 9-10 as well as our regular monitoring at $1.4-8.6 \mathrm{GHz}$. The $8.6 \mathrm{GHz}$ observation was reduced as described in $\mathrm{Ng}$ et al. (2008), and the 1.4-8.6 GHz flux monitoring observations and reduction are as described in G. Zanardo et al. (2009, in preparation; see also Manchester et al. 2002).

\section{RESULTS AND DISCUSSION}

\subsection{Flux Density and Spectral Index of the Remnant}

A flux density estimate was obtained for the naturally weighted (robust $=2.0$ ) 2008 April and October images. The amplitudes and phases of the April observation were affected by prevailing weather conditions, making the 2008 April 25 flux density estimate unreliable. A flux density of $27 \pm 6 \mathrm{mJy}$ was measured for the combined October image by imaging using natural weighting and taking a cut $6 \sigma$ above an rms noise of $60 \mu \mathrm{Jy} \mathrm{beam}^{-1}$. The flux density of the $1.4,2.4,4.8$, and $8.6 \mathrm{GHz} 2008$ October monitoring observations was measured to be $414 \pm 12,280 \pm 8,173 \pm 6$, and $112 \pm 6 \mathrm{mJy}$, respectively. This yields a spectral index $\alpha=-0.71 \pm 0.01$. When used to predict the 2008 October flux density at $36.2 \mathrm{GHz}$, the fitted power spectrum predicts a flux density of $41 \pm 6 \mathrm{mJy}$. The 2008 

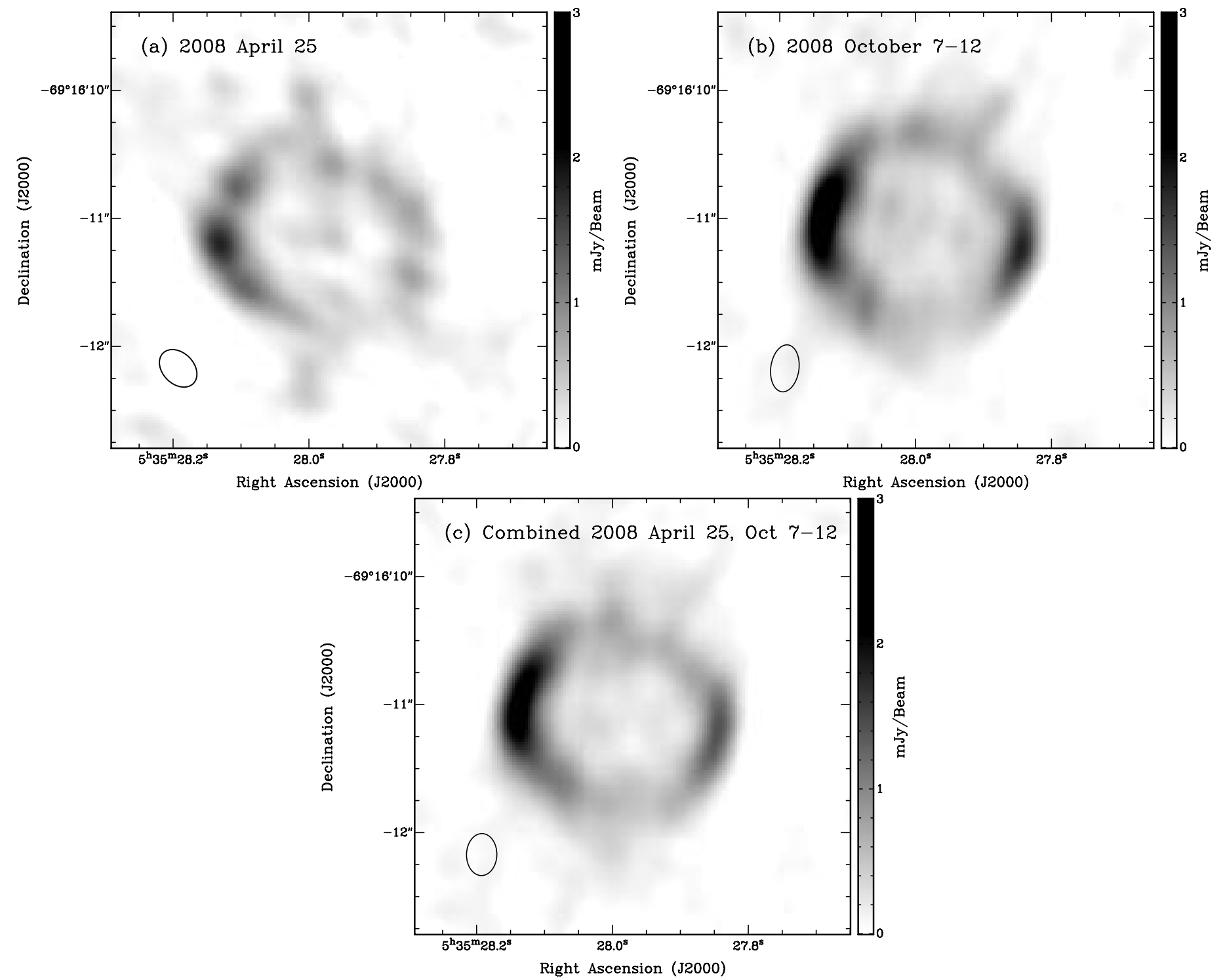

Figure 1. Continuum images of SN 1987A made with the ATCA at an average frequency of $36.2 \mathrm{GHz}$ and robust $=0.5$ weighting: (a) 2008 April 25 ; (b) 2008 combined October data; and (c) 2008 combined observations. The 2008 April, October, and combined images have an rms noise of $0.10,0.06$, and $0.06 \mathrm{mJy}^{\circ}$ beam ${ }^{-1}$, respectively. The linear gray scale on each image ranges from 0 to $3 \mathrm{mJy} \mathrm{beam}^{-1}$. The black ellipse at lower left is the beam as given in Table 2 .

October flux density of $27 \pm 6 \mathrm{mJy}$ therefore lies $1.3 \sigma$ lower than the power law fit to the other frequencies. If we include the 2008 October observation in the fit, we obtain a spectral index of $\alpha=-0.83$, where $S_{v} \propto v^{\alpha}$. A comparison of the fits is shown in Figure 2.

\subsection{Modeling in the Fourier Domain and a Limit on a Central Source}

We modeled the 2008 October 36.2 and $8.6 \mathrm{GHz}$ data in the Fourier domain using a truncated shell model described by $\mathrm{Ng}$ et al. (2008). The shell has a radius $R$ and a half-opening angle $\theta$ (i.e., $\theta=90^{\circ}$ and $0^{\circ}$ corresponds to a spherical shell and an equatorial ring, respectively), and it is inclined at $43^{\circ} .4$ south of the line of sight at a position angle -7.6 (or 7.6 clockwise) from north, as determined from optical observations (Pun 2007). We modeled the east-west asymmetry by a linear gradient in the equatorial plane, more details of the modeling can be referred to $\mathrm{Ng}$ et al. (2008). The best-fit model parameters at the two frequencies are shown in Table 3 and displayed graphically in the top row of Figure 3. Both models are roughly consistent, each with $30 \%$ greater flux in the eastern lobe. The 8.6 and $36.2 \mathrm{GHz}$ fitted shell widths do not agree at the $3 \sigma$ level. If this is a physical effect, it may be due to a positional dependency of the distribution of particle energies in the forward shock. The small shell width characteristic of the fits to low frequency radio emission may correspond to a proportionately larger population of newly swept up particles at the forward shock. However, shell thickness $\delta$ is a parameter that is not well constrained by the fit, as discussed in $\mathrm{Ng}$ et al. (2008) and demonstrated in Table 2 of that paper.

At $36.2 \mathrm{GHz}$, the model was subtracted from the data to produce a residual image as shown in Figure 4(c), alongside the imaged data and imaged model in Figures 4(a) and 4(b). Features present or absent in the fitted model appear as negative or positive components in the residual image. Within the remnant in the residual image, the highest positive peak is $+0.2 \pm$ $0.1 \mathrm{mJy}^{\text {beam }}{ }^{-1}$ and the lowest negative peak is $-0.2 \pm$ $0.1 \mathrm{mJy}$ beam $^{-1}$ with an average difference of zero. The 


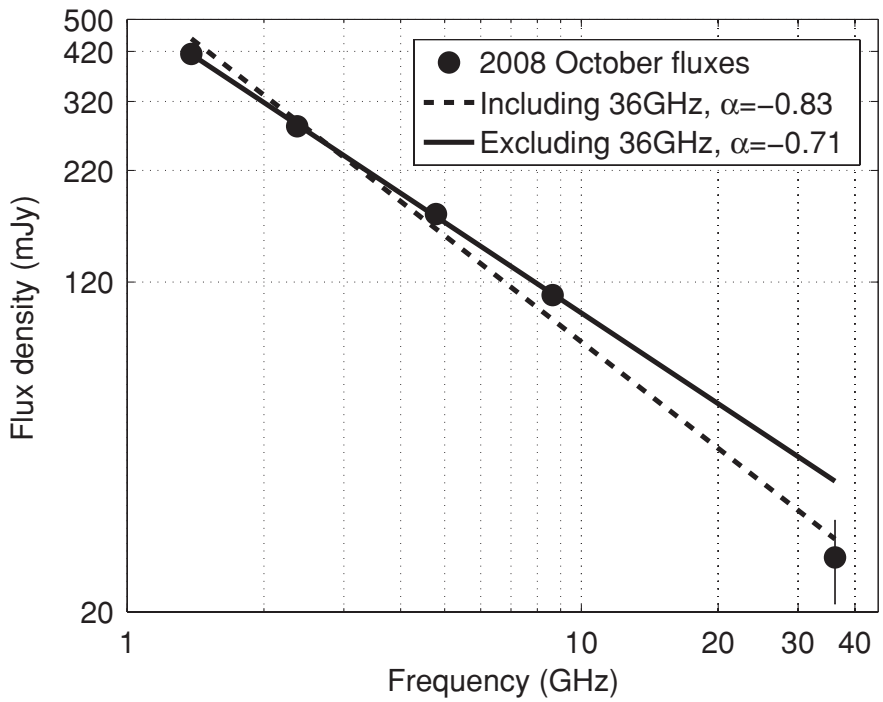

Figure 2. Spectral index of SN 1987A as determined by a power-law fit to the flux densities of the 2008 October monitoring observations both with and without the $36.2 \mathrm{GHz}$ observation. The spectral indices from the fit are $\alpha=-0.71$ for the solid line and $\alpha=-0.83$ for the dashed line.

Table 3

Best-fit Truncated-shell Models of the 2008 October $36.2 \mathrm{GHz}$ and 2008 October $8.6 \mathrm{GHz}$ Observations

\begin{tabular}{lcccc}
\hline \hline Frequency & $\begin{array}{c}\text { Flux Density } \\
f \\
(\mathrm{mJy})\end{array}$ & $\begin{array}{c}\text { Mean Shell Radius } \\
\mathrm{R} \\
(\mathrm{GHz})\end{array}$ & $\begin{array}{c}\text { Shell Width } \\
\delta\end{array}$ & $\begin{array}{c}\text { Half-opening } \\
\text { angle } \theta \\
(\%)\end{array}$ \\
\hline $36.2 \mathrm{GHz}$ & 32.7 & $0.850 \pm 0.060$ & $16 \pm 4$ & $29.2 \pm 2.0$ \\
$8.6 \mathrm{GHz}$ & 108.0 & $0.892 \pm 0.002$ & $0.04 \pm 2.6$ & $35.6 \pm 0.6$ \\
\hline
\end{tabular}

amplitude of the peak, the maximum difference between the real and model image, represents an upper limit on the flux density from a possible central source. A more quantitative measurement was made by adding an additional point source to the truncated torus model and fitting the model to the 2008 October uv-data at $36.2 \mathrm{GHz}$. The resulting flux density of the fitted point source in the model is $0.3 \pm 0.2 \mathrm{mJy}$, which is consistent with the above estimate. It is also consistent with the $0.3 \mathrm{mJy}$ upper limit at $9 \mathrm{GHz}$, estimated in $\mathrm{Ng}$ et al. (2008). Possible reasons for this extra point source flux include: an energized population of accelerated electrons due to projected shell emission at high latitudes away from the equatorial ring, a source within the central region of the remnant, noise, or possibly phase errors in the $36.2 \mathrm{GHz}$ image. Assuming $0.3 \mathrm{mJy}$ as the flux density of a possible central source, we estimate a $36 \mathrm{GHz}$ spectral luminosity of $1 \times 10^{14} \mathrm{~W} \mathrm{~Hz}^{-1}$ at a distance of $51.4 \mathrm{kpc}$ (Panagia 2003). This value is significantly under-luminous when compared to some central sources of other known SN remnants. Page et al. (2007) obtain a flux density of $322 \mathrm{Jy}$ at $33 \mathrm{GHz}$ and $299 \mathrm{Jy}$ at $40 \mathrm{GHz}$ from WMAP observations of the Crab Nebula. Assuming an interpolated flux density of $310 \mathrm{Jy}$ for $36.2 \mathrm{GHz}$ gives a luminosity for the Crab of $1.5 \times 10^{17} \mathrm{~W} \mathrm{~Hz}^{-1}$ at a distance of $2 \mathrm{kpc}$ (Trimble 1973). Observations at $22 \mathrm{GHz}$ of the young remnant of SN 1986J (Bietenholz \& Bartel 2008) show a flux density of $4 \mathrm{mJy}$ in a central region of flat spectral index. Extrapolated to $36.2 \mathrm{GHz}$, this corresponds to a flux density of $3 \mathrm{mJy}$ or a luminosity of $4 \times 10^{19} \mathrm{~W} \mathrm{~Hz}^{-1}$ at a distance of $10 \mathrm{Mpc}$.

\subsection{Spectral Index Image}

SN 1987A has been "classified" as a Type IIP SN with a progenitor mass of around 17-20 solar masses (Saio et al. 1988). We know from neutrino observations that a large number of neutrons were formed in the core collapse. However, it is still uncertain if a neutron star survived the explosion. Searches for an associated pulsar have been unsuccessful so far (Manchester 2007), although they only rule out the existence of a relatively high-luminosity pulsar. Another possible way to find evidence of a surviving neutron star is to look for a pulsar wind nebula (PWN). PWNe are clouds of non-thermally radiating particles that are excited by an enclosed compact central source. PWNe have been detected in about 10 percent of all known core collapse SN remnants (Kaspi \& Helfand 2002), with the Crab nebula and 3C58 being some of the best-known examples. In the radio such nebulae are characterized by polarized synchrotron emission and typically have a flat spectral index of ranging between -0.3 and 0 (Gaensler \& Slane 2006). Detection of flat spectral index emission near the explosion site of SN 1987A, as was the case with SN 1986J (Bietenholz et al. 2004), would be a good indication that a neutron star has survived the explosion. A search for a possible central flat spectrum source was undertaken by generating a spectral index image using the 2008 October data at $36.2 \mathrm{GHz}$ and the 2008 October data at $8.6 \mathrm{GHz}$ (discussed in Section 2). As the synthesized beam for the $8.6 \mathrm{GHz}$ data is $00^{\prime \prime} 4$, the $36.2 \mathrm{GHz}$ data were re-imaged with a 0 ". 4 restoring beam using the MAXEN algorithm instead of CLEAN for consistency with the $8.6 \mathrm{GHz}$ image. Both the $8.6 \mathrm{GHz}$ and the re-imaged $36.2 \mathrm{GHz}$ images are shown in the middle row of Figure 3. As a precaution against possible false spectral index information generated by the imaging process, we also produced a comparison spectral index image made from the Fourier models in the top row of Figure 3. The models were inserted into the visibility data with a suitable position offset to locate it at the same angular distance from the phase center but on the opposite side of the phase center from the remnant. Following imaging and deconvolution, the resulting model images, shown in the bottom row of Figure 3, have approximately the same noise, flux density, and restoring beam characteristics as their real counterparts. In Figure 5 we present the two spectral index images. Figure 5(a) was formed by comparing the 2008 October $8.6 \mathrm{GHz}$ and re-imaged 2008 October $36.2 \mathrm{GHz}$ observations, and Figure 5(b) was formed by comparing the 2008 October $8.6 \mathrm{GHz}$ and 2008 October $36.2 \mathrm{GHz}$ model images. The real spectral index image has been masked using the same mask used to determine the flux of the 2008 October CLEAN image at $36.2 \mathrm{GHz}$. A similar mask has also been applied to the model spectral index image.

Both spectral index images share the same broad characteristics, regions of spectral index, $\alpha \approx-0.8$ at the two lobes and regions of steep spectral index, $\alpha \approx-1.5$ in the interior. There are two notable flat spectral index features in the real image. The feature at the northern rim is caused by the spurious northsouth extensions mentioned in Section 2. The feature around 0!'2 east of the progenitor position, which has a maximum spectral index of $\alpha \approx-0.7 \pm 0.3$ and encompasses an area of approximately one beam (width $0{ }^{\prime \prime} 4$ ). The position of the flattest spectral index in this feature is $05^{\mathrm{h}} 35^{\mathrm{m}} 27^{\mathrm{s}} .991,-69^{\circ} 16^{\prime} 11^{\prime \prime} .13$ (J2000) with around 50 mas positional uncertainty. However, a smaller flat spectral index feature is also present in the model counterpart. This feature is largely due to the imaging process rather than noise or differences between the models. Follow-up 

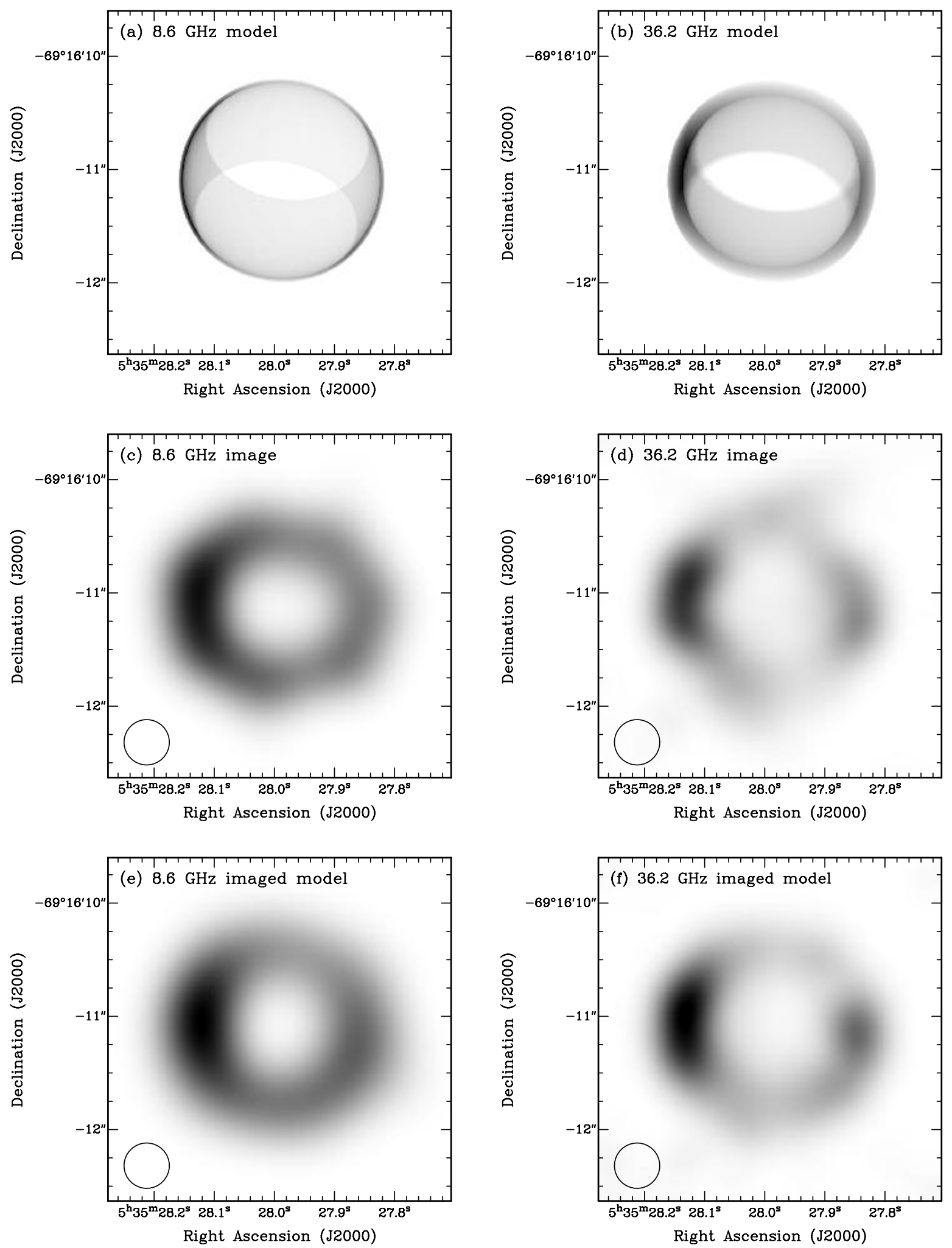

Figure 3. Fourier models and images from the 2008 October observation at $8.6 \mathrm{GHz}$ and $36.2 \mathrm{GHz}$. The left column contains $8.6 \mathrm{GHz}$ images, the right column $36.2 \mathrm{GHz}$ images obtained using the same MAXEN deconvolution algorithm. In the top row are the truncated-shell models, the middle row contains the real images. The bottom row contains model images formed by inserting the model visibility data, generated from the top row, into the observed visibility data from the middle row and running them through the same imaging pipeline. The model images (bottom row) thus include noise and have the same uv coverage as the real observations (middle row). All images have been restored using an identical $0{ }^{\prime} .4$ beam and have a linear gray scale that ranges from $0-13 \mathrm{mJy}$ for the $8.6 \mathrm{GHz}$ images and $0-3.5 \mathrm{mJy}$ for the $36.2 \mathrm{GHz}$ images respectively.

observations will be needed to determine if the feature in the real spectral index images corresponds to an actual source of emission in the central region emission not fitted by our Fourier models. 

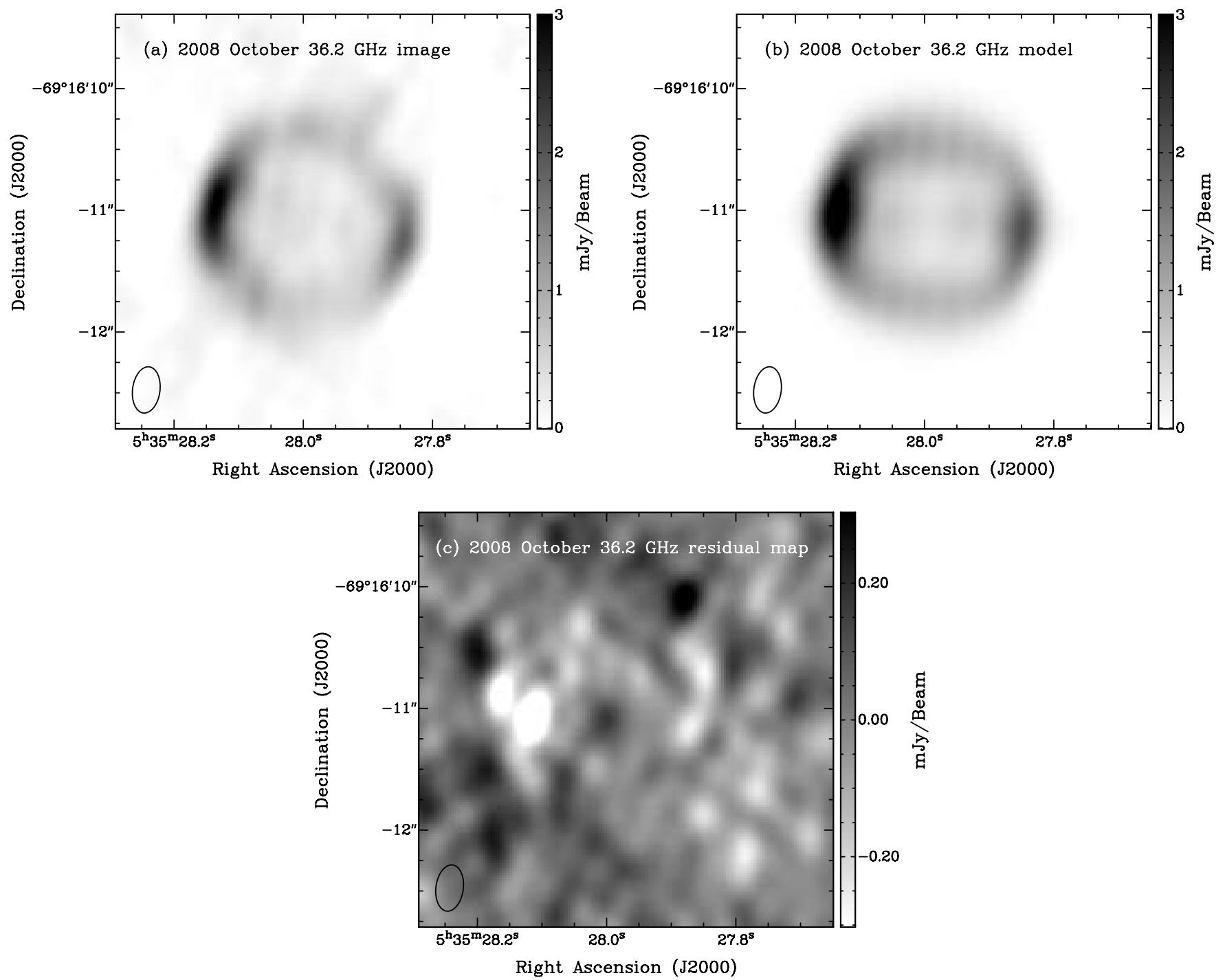

Figure 4. (a) 2008 October $36.2 \mathrm{GHz}$ image of SN 1987A; compared with (b), the model from the Fourier-domain fitting (Ng et al. 2008); and (c) the imaged residual image between the image and model. The real and model mages are displayed on a linear gray-scale ranging from 0 to $4 \mathrm{mJy}_{\text {beam }}^{-1}$, and the residual image has a gray scale ranging $\pm 0.3 \mathrm{mJy}_{\text {beam }}^{-1}$. The rms noise of the residual image is estimated to be around $90 \mu \mathrm{Jy}$.
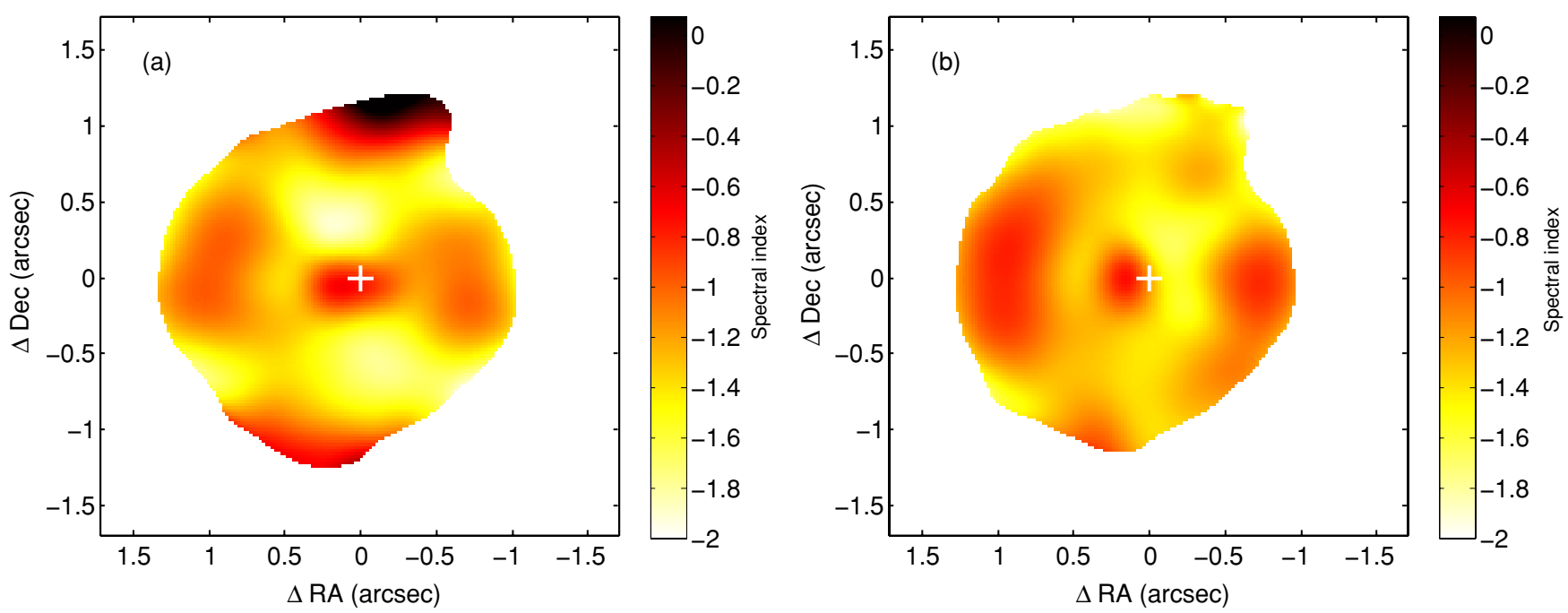

Figure 5. Spectral index images formed by comparing the super-resolved 2008 October $8.6 \mathrm{GHz}$ and the re-imaged $2008 \mathrm{October} 36.2 \mathrm{GHz}$ images of SN $1987 \mathrm{~A}$ with the same MAXEN deconvolution algorithm and 0!'4 restoring beam. Panel (a) is the real spectral index image and panel (b) is the model spectral index image generated from the imaged Fourier models. The progenitor position is shown as a white cross in each image. 
Table 4

Parameters of the Flux-weighted and Flux Un-weighted Elliptical Fits

\begin{tabular}{lccccc}
\hline \hline Weighting & $\begin{array}{c}\text { RA Offset }^{\mathrm{a}} \\
(\mathrm{mas})\end{array}$ & $\begin{array}{c}\text { Dec Offset }^{\mathrm{a}} \\
(\mathrm{mas})\end{array}$ & $\begin{array}{c}\text { Semimajor Axis } \\
\left({ }^{\prime \prime}\right)\end{array}$ & $\begin{array}{c}\text { Semiminor Axis } \\
\left({ }^{\prime \prime}\right)\end{array}$ & $\begin{array}{c}\text { Position Angle }^{\mathrm{b}} \\
\left({ }^{\circ}\right)\end{array}$ \\
\hline Weighted & $+72 \pm 48$ & $-37 \pm 46$ & 0.79 & 0.68 & 74.7 \\
None & $+79 \pm 48$ & $-32 \pm 46$ & 0.80 & 0.66 & 70.6 \\
\hline
\end{tabular}

Notes.

a Offsets are the angular distances of the fitted ellipse centroids from the predicted position of $05^{\mathrm{h}} 35^{\mathrm{m}} 27^{\mathrm{s}} .975,-69^{\circ} 16^{\prime} 11^{\prime \prime}$.08 (J2000).

b Position angle is defined north through east.

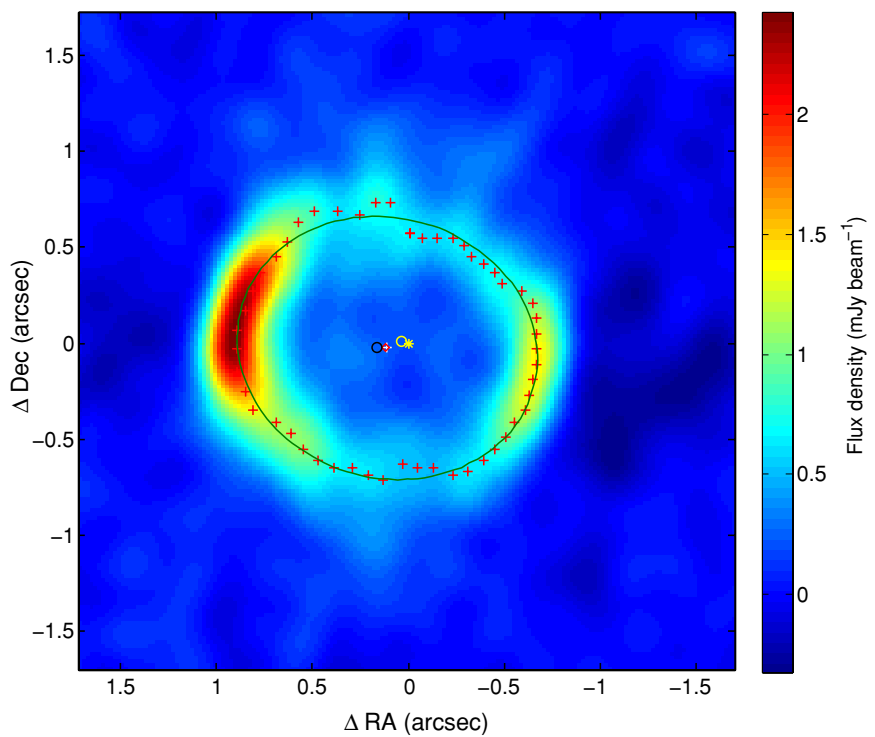

Figure 6. Results from the ellipse fits to the morphology of the combined 2008 October and 2008 April $36.2 \mathrm{GHz}$ observations. The green ellipse drawn atop the image is the flux-weighted ellipse fit to the red crosses returned by the ridge-following algorithm. The centroid of the green ellipse is shown as a white cross almost hidden under the red diamond which represents the centroid of the flux-unweighted ellipse fit. The yellow star is the measured optical position of the progenitor (Reynolds et al. 1995); this moves to the position of the small yellow circle when the proper motion of the remnant and the LMC is accounted for. The black circle represents the position of the flat spectral index feature.

\subsection{Polarization and Magnetic Field Direction}

Magnetic field direction in SN remnants can be divided into three categories: tangled, radial and tangential. In the atlas of SN remnants compiled by Milne (1987, 27 out of the 70 remnants surveyed showed polarization. The absence of polarization in the presence of synchrotron emission indicates a tangled magnetic field and/or extreme rotation measures along the line of sight. Of those remnants which did exhibit polarization, such as Cassiopeia A and Tycho's remnant, most under 10,000 years old had radial magnetic field directions across the shock front. Rayleigh-Taylor (R-T) instabilities have been implicated as the mechanism for the radial stretching of the magnetic field in young SN remnants; however, magnetohydrodynamical simulations have yet to produce R-T instabilities that reach across the forward shock (Blondin \& Ellison 2001). It is anticipated that higher shock compression ratios generated by the influence of shock-accelerated particles will enable the generation of radial magnetic fields that cross the shock (Schure et al. 2008). Older SN remnants such as Vela exhibit a tangential magnetic field, interpreted as the accumulation of an interstellar magnetic field at the ageing blast front.
A search was made for linear and circularly polarized emission in the remnant of SN 1987A by constructing polarization intensity images with natural weighting. No persistent linear polarization in Stokes-Q was detected above a $3 \sigma$ threshold of 0.6 and $0.24 \mathrm{mJy} \mathrm{beam}^{-1}$ for the 2008 April and 2008 October observations. A similar technique was employed to search for circular polarization in Stokes-V; however, the same null result was obtained for a $3 \sigma$ threshold of 0.6 and $0.24 \mathrm{mJy} \mathrm{beam}^{-1}$. Possible causes for the de-polarization of the radio emission include a tangled magnetic field in the emitting region and/or extreme Faraday rotation measures on the order of $\approx 50000 \mathrm{rad} \mathrm{m}^{-2}$.

\subsection{Radial Profile and Astrometry}

The optical position of the SN at day 1278, as measured by a frame-tie to the Hipparcos and VLBI reference frames is $05^{\mathrm{h}} 35^{\mathrm{m}} 27^{\mathrm{s}} .968,-69^{\circ} 16^{\prime} 11^{\prime \prime} .09$ (J2000), with an error of 30 mas in each coordinate (Reynolds et al. 1995). At day 7899, corresponding to 2008 October 9 , this position will have moved due to the proper motion of the remnant. The proper motion of the LMC according to Kallivayalil et al. (2006) is $+2.03 \pm 0.08$ mas $\mathrm{yr}^{-1}$ east and $+0.44 \pm 0.05$ mas $\mathrm{yr}^{-1}$ north. The remnant also experiences a $0.3 \pm 0.02$ mas $\mathrm{yr}^{-1}$ motion north due to its estimated $65 \pm 5 \mathrm{~km} \mathrm{~s}^{-1}$ bulk rotation speed around the LMC (Kim et al. 1998) at a distance of approximately $5 \mathrm{kpc}$ from the LMC's kinematic center (assuming a distance of $50 \mathrm{kpc}$ to SN 1987A). If corrected for both effects, then the progenitor should, at day 7899 , be situated $37 \pm 1.5$ mas east and $13 \pm 1.3$ mas north of Reynold's position, assuming an LMC distance of $50 \mathrm{kpc}$. This predicted position corresponds to the coordinates of $05^{\mathrm{h}} 35^{\mathrm{m}} 27^{\mathrm{s}} .975,-69^{\circ} 16^{\prime} 11^{\prime \prime} .08$ (J2000) with an uncertainty of 30 mas in each coordinate. We tested this prediction by fitting an ellipse to points that make up a ridgeline of the radio ring in the combined 2008 April-October $36.2 \mathrm{GHz}$ image. Points along the ridgeline in the radio image were located by propagating rays away from the original progenitor position. The point of maximum flux density along each propagated ray was determined to be part of the ridgeline. We fitted an ellipse to both flux-weighted and non-flux-weighted ridge line points and tabulated the fitted ellipsoids in Table 4. We see that fitting an ellipse to the $36.2 \mathrm{GHz}$ October data in the image plane produces a semimajor axis that is slightly smaller but consistent with the mean radius obtained by fitting a truncated-shell model to visibility data in the uv plane.

In Figure 6 the flux-weighted ellipse and ridgeline points are overlaid atop the combined 2008 April-October $36.2 \mathrm{GHz}$ image along with the two fitted ellipse centroids and the original progenitor position. The flux-weighted ellipse centroid was located at $05^{\mathrm{h}} 35^{\mathrm{m}} 27^{\mathrm{s}} .987,-69^{\circ} 16^{\prime} 11^{\prime \prime} .115$ (J2000) and the un-weighted centroid was located at 



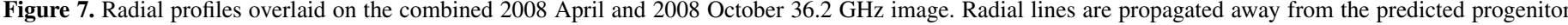
position in (a) and their radial distances are measured in (b). The positive distances measured in (b) correspond to rays propagating through the left half of (a).

$05^{\mathrm{h}} 35^{\mathrm{m}} 27^{\mathrm{s}} .990,-69^{\circ} 16^{\prime} 11^{\prime \prime} .110$ (J2000). Because we have used the VLBI position of the phase calibrator 0530-727 from Reynolds et al. (1995), the estimated positional uncertainty of the fitted centroids above is about 30 mas in each coordinate with an additional $\approx 15-17$ mas uncertainty in the fit. As shown in Figure 6, the offset of the flux-weighted centroid is $72 \pm$ 48 mas east and $37 \pm 45$ mas south of the predicted position, and the flux-unweighted centroid is $79 \pm 48$ mas east and $32 \pm$ 46 mas south of the predicted position. Given that the positional uncertainty in the predicted position is about 30 mas, we conclude that the two fitted centroids and the predicted position all lie within $1 \sigma$ of each other. If a hydrodynamical effect following the explosion is the cause of the centroid offset from the predicted position, we estimate that the (day 7899) upper limit for any departure from symmetry for the eastern lobe, estimated as approximately twice the offset plus uncertainty, is at most 174 mas east and 64 mas south. The position of the possible flat spectral index feature in Figure 5(a) was offset 124 mas east and 32 mas south of the predicted position, which places it within 60 mas of the fitted centroids.

Radial profiles of the remnant were made by propagating rays away from the averaged position of the ellipse centroid (obtained from the ridge-finding algorithm) and plotting the resulting profiles, shown in Figure 7 . In particular, note that the flux density within the projected radio ring on the image plane is always greater than $6 \sigma$ above the rms noise of $0.06 \mathrm{mJy} \mathrm{beam}^{-1}$, which indicates that emission is being produced along the line of sight, either from high latitude regions of the expanding shell, or from within the central region of the nebula itself.

\subsection{Comparison with Observations at Other Wavelengths}

In order to see how the 2008 October $8.6 \mathrm{GHz}$ and 2008 October $36.2 \mathrm{GHz}$ images compared with observations at other wavelengths we obtained optical HST and X-ray Chandra images from their respective public archives. The HST image was taken on 2006 December 6 using filter F625W in ACS/HRC configuration (data set id\# J9OT07070). The Chandra X-ray image was a $0.5-8.0 \mathrm{keV}$ ACIS image from an observation taken on 2008 July 9-11. The X-ray image was reprocessed following the procedure described in Park et al. (2006). We removed the pixel randomization and applied the "subpixel resolution" technique (Tsunemi et al. 2001) to further improve the effective spatial resolution of the image. It was then deconvolved with the Lucy-Richardson algorithm (Richardson 1972; Lucy 1974) using a monochromatic PSF of $1.5 \mathrm{keV}$ (2.5 keV for the hard band) generated by the Chandra Ray Tracer and MARX. The results were smoothed by a Gaussian of 0'. 1 FWHM. Shown in Figure 8 is a comparison between the HST, 36.2 GHz ATCA, 8.6 GHz ATCA, and Chandra X-ray images. The coordinates of the HST and Chandra images have been shifted such that the optical and X-ray rings are centered on the 2008 October $36.2 \mathrm{GHz}$ radio ring with an uncertainty of roughly 50 mas in each coordinate; it also implies the central optical nebulosity aligns with the flat spectrum radio component. However, this step precludes us from making further judgements about an asymmetry in the radio expansion with respect to the optical ring. One thing that is interesting about this plot, however, is that the radio images are more circular than the optical and X-ray. If we apply the ridge-following algorithm to the flux-unweighted optical ring in the HST image, the width of the fitted ellipse semimajor axis is 0.78 and the semiminor axis is 0.57 with about 40 mas uncertainty in each axis. Compared to the radio ring of size $0.79 \times 0$ '. 68 with similar uncertainty, the optical ring is slightly more elliptical. The difference in ellipticity can be explained by the difference in location at which the different types of emission are generated. Non-thermal radio emission is produced as the result of stochastic acceleration of particles at a shock front, and is primarily dependent on density for particle injection into the shock. Thermal X-rays are produced by material heated by the shock and is dependent on both density and temperature of the heated material. Hence we would expect 

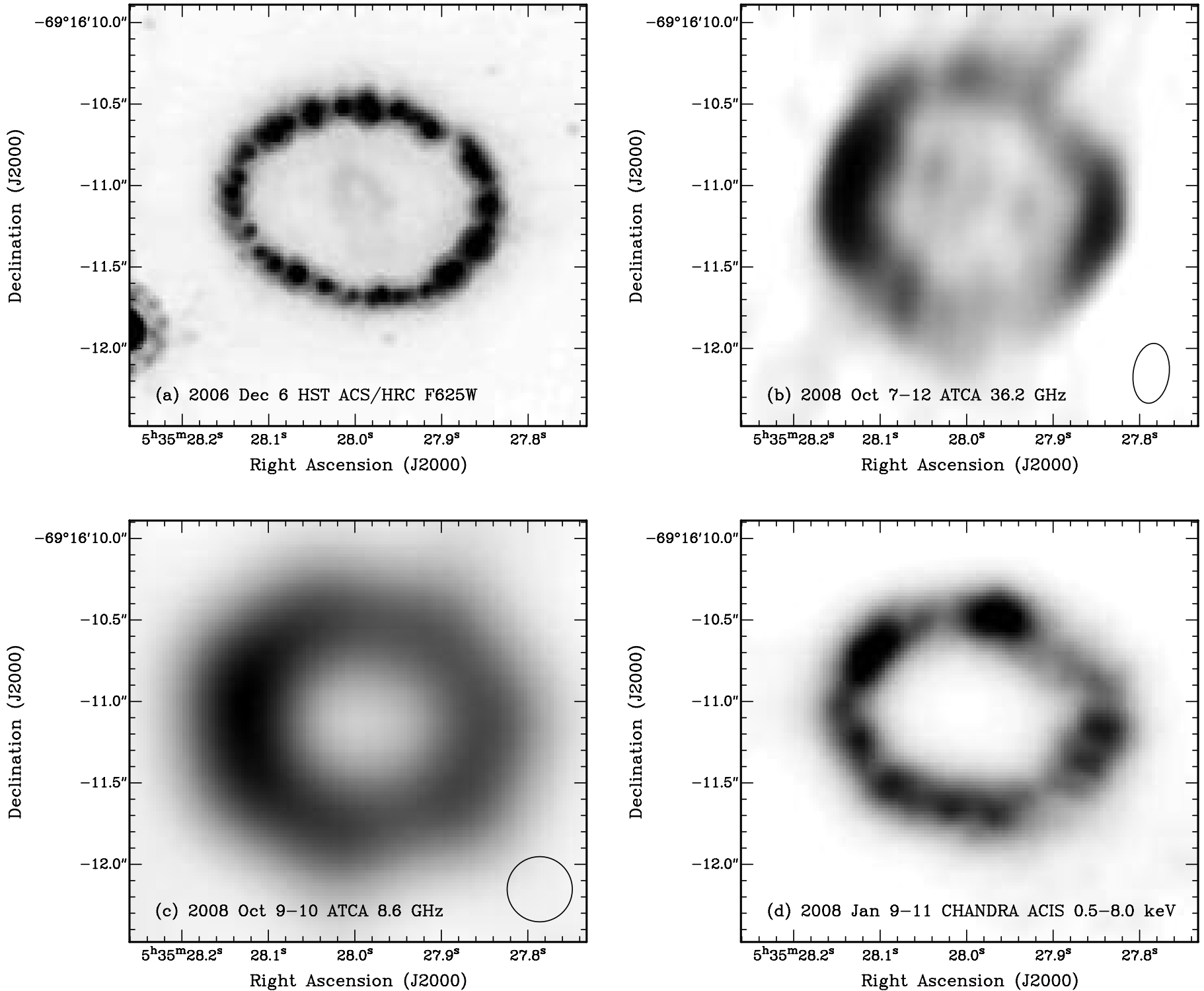

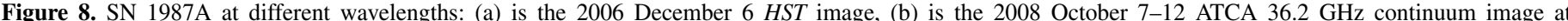

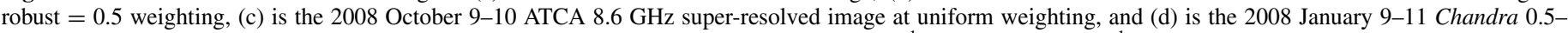

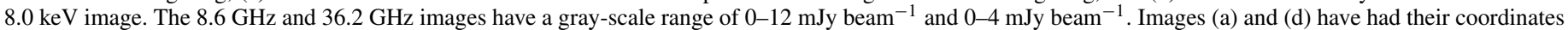
shifted to coincide with the radio images as in Figure 9. The linear gray scale on each image has been stretched to highlight intrinsic low brightness features.

radio emission to trace shock fronts to higher latitudes above the equatorial plane and X-ray and optical images to trace the higher density of heated material in the equatorial ring.

In Figure 9 the $H S T$ and Chandra images have been overlaid with the radio contours from the $36.2 \mathrm{GHz}$ radio image. From the peaks in the radio contours it is evident that the forward shock is only slightly larger than the 0 ' 8 optical ring. The truncatedshell models fitted to the 2008 October data give a more accurate shell radius of $0{ }^{\prime} \cdot 85 \pm 00^{\prime} 06$ for the $36 \mathrm{GHz}$ data which is larger but still consistent. However, the radius measured at $8.6 \mathrm{GHz}$ is $00^{\prime} 892 \pm 0 . \prime 002$, which suggests that the forward shock has almost completed its transit of the optical ring.

\section{CONCLUSIONS}

In this paper we have presented results from the $36.2 \mathrm{GHz}$ extension of the monitoring observations of SN 1987A performed in 2008 April and October. The October observation was con- ducted under conditions of good atmospheric stability. A flux density of $27 \pm 6 \mathrm{mJy}$ was obtained which allows us to derive a spectral index of $\alpha \approx-0.83$ when fitted along with flux densities from the lower frequency monitoring observations. The Fourier modeling technique of $\mathrm{Ng}$ et al. (2008) was employed to produce a model fit of the torus that is consistent with the $8.6 \mathrm{GHz}$ image. A comparison of the $36.2 \mathrm{GHz}$ image with the $8.6 \mathrm{GHz}$ image at similar resolution was used to obtain a spectral index image that showed a region of flatter spectral index about 0.2 east of the progenitor. Simulations suggest a component of this may be an artifact of the imaging process. However, follow-up observations are suggested to determine if the remaining component is real central region emission not fitted by our Fourier models. Higher sensitivity observations with the newly commissioned Compact Array Broadband Backend (CABB) will help further clarify this feature. An elliptical fit to the $36.2 \mathrm{GHz}$ radio morphology shows that the centroid of the remnant is within positional accuracy of the progenitor's 


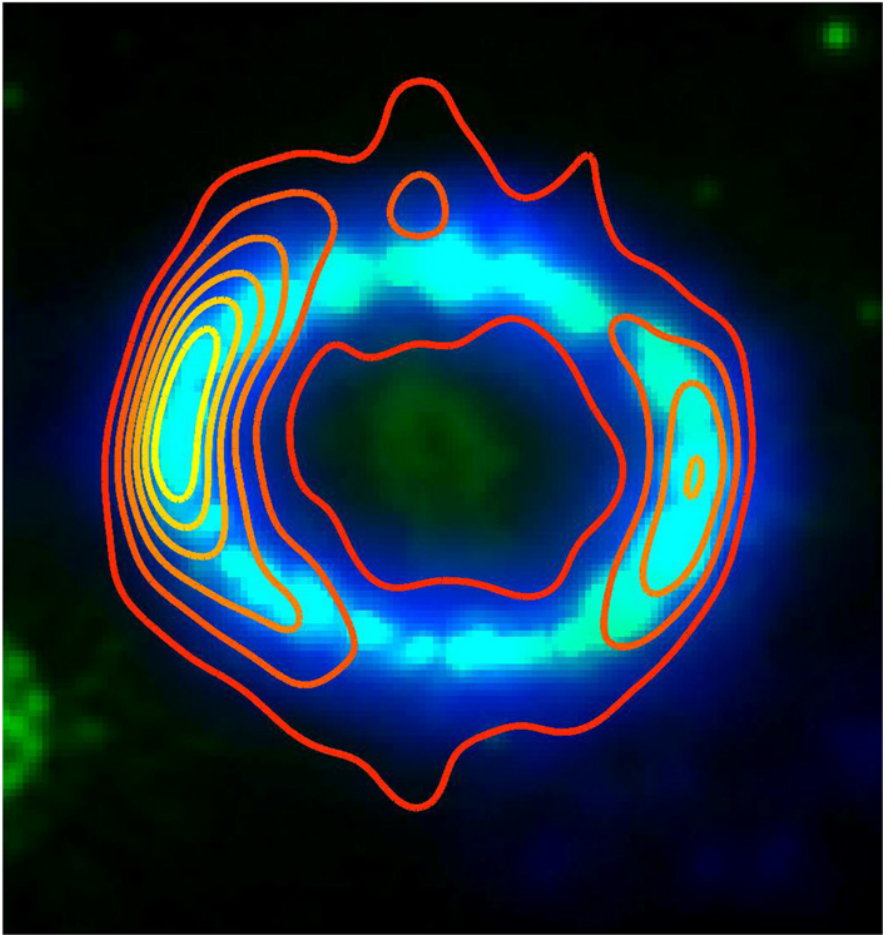

Figure 9. Overlay of the combined HST 2006 December 6 optical (green), Chandra 2008 January 9-11 X-ray (blue), and ATCA 2008 October $36.2 \mathrm{GHz}$ radio images (orange-yellow contours) formed by shifting the optical and X-ray coordinate systems to center on the radio ring from the 2008 October $36.2 \mathrm{GHz}$ radio image at robust $=0.5$ weighting. Radio contours are at 14 (orange), 30, 40, 60,70 , and $85 \%$ (yellow) of the maximum at $2.4 \mathrm{mJy}^{\text {beam }}{ }^{-1}$. The outermost contour and the contour within the optical ring are at the same $14 \%$ level.

location when corrected for the proper motion of the LMC and the motion of SN 1987A within the LMC. The fit is also located within positional uncertainty of the flat spectral index feature. Comparisons between radio and X-ray images show that the remnant is more spherical in the radio, which can be explained by two different emission mechanisms at work. From the Fourier models and comparisons to the optical emission we conclude that the forward shock in the remnant is completing its transit of the optical ring.

We thank Bjorn Emonts and Robin Wark for their assistance with our observations, Andre Fletcher for useful discussions on radio reduction, and Brian Reville and John Kirk for their insightful comments on particle acceleration at shock fronts. This research has made use of data obtained from the Chandra Data Archive. Some of the data presented in this paper were obtained from the Multimission Archive at the Space Telescope Science Institute (MAST). STScI is operated by the Association of Universities for Research in Astronomy, Inc., under NASA contract NAS5-26555. Support for MAST for non-HST data is provided by the NASA Office of Space Science via grant NAG57584 and by other grants and contracts. C.-Y.N. and B.M.G. acknowledge the support of the Australian Research Council through grant FF0561298.

\section{REFERENCES}

Ball, L., Crawford, D. F., Hunstead, R. W., Klamer, I., \& McIntyre, V. J. 2001, ApJ, 549, 599
Bietenholz, M. F., \& Bartel, N. 2008, Adv. Space Res., 41, 424

Bietenholz, M. F., Bartel, N., \& Rupen, M. P. 2004, Science, 304, 1947

Bionta, R. M., Blewitt, G., Bratton, C. B., Caspere, D., \& Ciocio, A. 1987, Phys. Rev. Lett., 58, 1494

Blondin, J. M., \& Ellison, D. C. 2001, ApJ, 560, 244

Blondin, J. M., \& Lundqvist, P. 1993, ApJ, 405, 337

Briggs, D. S. 1995, BAAS, 27, 1444

Brooks, K. 2007, ATCA@7mm, Sydney, NSW: CSIRO (ATNF), http://www. atnf.csiro.au/observers/docs $/ 7 \mathrm{~mm} /$

Chevalier, R. A., \& Dwarkadas, V. V. 1995, ApJ, 452, L45

Clark, B. G. 1980, A\&A, 89, 377

Gaensler, B. M., Manchester, R. N., Staveley-Smith, L., Tzioumis, A. K., Reynolds, J. E., \& Kesteven, M. J. 1997, ApJ, 479, 845

Gaensler, B. M., \& Slane, P. O. 2006, Annu. Rev. Astron. Astrophys., 44, 17

Gaensler, B. M., Staveley-Smith, L., Manchester, R. N., Kesteven, M. J., Ball, L., \& Tzioumis, A. K. 2007, AIP Conf. Proc., 937, 86

Hirata, K., Kajita, T., Koshiba, M., Nakahata, M., \& Oyama, Y. 1987, Phys. Rev. Lett., 58, 1490

Högbom, J. A. 1974, A\&AS, 15, 417

Janka, H.-Th. 1997, arXiv:astro-ph/9709013

Jauncey, D. L., Kemball, A., Bartel, N., Shapiro, I. I., Whitney, A. R., Rogers, A. E. E., Preston, R. A., \& Clark, T. A. 1988, Nature, 334, 412

Kallivayalil, N., van der Marel, R. P., Alcock, C., Axelrod, T., Cook, K. H., Drake, A. J., \& Geha, M. 2006, ApJ, 638, 772

Kaspi, V. M., \& Helfand, D. J. 2002, in ASP Conf. Ser. 271, Neutron Stars in Supernova Remnants, ed. P. O. Slane \& B. M. Gaensler (San Francisco, CA: ASP), 3

Kim, S., Staveley-Smith, L., Dopita, M. A., Freeman, K. C., Sault, R. J., Kesteven, M. J., \& McConnell, D. 1998, ApJ, 503, 674

Lucy, L. B. 1974, AJ, 79, 745

Luo, D. \& McCray, R. 1991, ApJ, 379, 659

Manchester, R. N. 2007, in AIP Conf. Proc. 937, SUPERNOVA 1987A: 20 YEARS AFTER: Supernovae and Gamma-Ray Bursters, ed. S. Immler, K. Weiler, \& R. McCray (Melville, NY: AIP), 134

Manchester, R. N., Gaensler, B. M., Staveley-Smith, L., Kesteven, M. J., \& Tzioumis, A. K. 2005, ApJ, 628, L131

Manchester, R. N., Gaensler, B. M., Wheaton, V. C., Staveley-Smith, L., Tzioumis, A. K., Bizunok, N. S., Kesteven, M. J., \& Reynolds, J. E. 2002, PASA, 19, 207

Martin, C. L., \& Arnett, D. 1995, ApJ, 447, 378

McMaster, M., \& Biretta, J. 2008, WFPC2 Instrument Handbook (10th ed.; Baltimore, MD: Space Telescope Science Institute), 338

Michael, E., et al. 2003, ApJ, 593, 809

Milne, D. K. 1987, Aust. J. Phys., 40, 771

Murphy, T., Gaensler, B. M., \& Chatterjee, S. 2008, MNRAS, 389 L23

Ng, C. Y., Gaensler, B. M., Staveley-Smith, L., Manchester, R. N., Kesteven, M. J., Ball, L., \& Tzioumis, A. K. 2008, arXiv:0805.4195v1

Page, L., et al. 2007, ApJS, 170, 335

Panagia, N. 2003, arXiv:astro-ph/0309416v1

Park, S., Zhekov, S. A., Burrows, D. N., Racusin, J. L., McCray, R., \& Borkowski, K. J. 2006, in Proceedings of The X-ray Universe 2005, ed. A. Wilson (ESA SP-604; Noordwijk: ESA Publications Division), 335

Plait, P. C., Lundqvist, P., Chevalier, R. A., \& Kirshner, R. P. 1995, ApJ, 439 , 730

Podsiadlowski, P., Morris, T. S., \& Ivanova, N. 2007, in AIP Conf. Proc. 937, SUPERNOVA 1987A: 20 YEARS AFTER: Supernovae and Gamma-Ray Bursters, ed. S. Immler, K. Weiler, \& R. McCray (Melville, NY: AIP), 125

Pun, C. S. J. 2007, in AIP Conf. Ser. 937, Supernova 1987A: 20 Years After: Supernovae and Gamma-Ray Bursters, ed. S. Immler, K. Weiler, \& R. McCray (Melville, NY: AIP), 171

Reynolds, J. E., et al. 1995, A\&A, 304, 116

Reynolds, S. P., Borkowski, K. J., Green, D. A., Hwang, U., Harrus, I., \& Petre, R. 2008, ApJ, 680, L41

Richardson, W. H. 1972, J. Opt. Soc. Am., 62, 55

Saio, H., Nomoto, K., \& Kato, M. 1988, ApJ, 331, 388

Sault, R. J., Teuben, P. J., \& Wright, M. C. H. 1995, in ASP Conf. Ser. 77 , Astronomical Data Analysis Software and Systems IV, ed. R. A. Shaw, H. E. Payne, \& J. J. E. Hayes (San Francisco, CA: ASP), 433

Schure, K., Vink, J., Achterberg, B., \& Keppens, R. 2008, arXiv:0810.5150v1 Staveley-Smith, L., Gaensler, B. M., Manchester, R. N., Ball, L., Kesteven, M. J., \& Tzioumis, A. K. 2007, in AIP Conf. Proc. 937, SUPERNOVA 1987A: 20 YEARS AFTER: Supernovae and Gamma-Ray Bursters, ed. S. Immler, K. Weiler, \& R. McCray (Melville, NY: AIP), 96 
Staveley-Smith, L., et al. 1992, Nature, 355, 147

Sugerman, B. E. K., Crotts, A. P. S., Kunkel, W. E., Heathcote, S. R., \& Lawrence, S. S. 2005, ApJS, 159, 60

Tanaka, T., \& Washimi, H. 2002, Science, 296, 321

Taylor, G., Carrilli, C., \& Perley, R. (eds.) 1999, in ASP Conf. Ser. 180, Synthesis Imaging in Radio Astronomy (San Francisco, CA: San Francisco: ASP)
Tingay, S. J., et al. Proc. 8th Int. e-VLBI Workshop, Proceedings of Science Trimble, V. 1973, PASA, 85, 579

Tsunemi, H., Mori, K., Miyata, E., Baluta, C., Burrows, D. N., Garmire, G. P., \& Chartas, G. 2001, ApJ, 554, 496

Turtle, A. J., Campbell-Wilson, D., Bunton, J. D., Jauncey, D. L., \& Kesteven, M. J. 1987, Nature, 327, 38 